\title{
¿Satisfacción por vocación?: La influencia del tipo de trabajo en la satisfacción laboral
}

Ángeles Jiménez Peinado, Miriam Pérez Martínez, Isabel Pinel Jiménez, Julia Sánchez García y Laura Villanueva Moya. Universidad de Granada

Recepción: 2 de mayo de 2016 | Revisión: 4 de mayo de 2016 | Aceptado: 9 mayo de 2016

Correspondencia: juliasanchezg@correo.ugr.es

Citar: Jiménez Peinado, A., Pérez Martínez, M., Pinel Jiménez, I., Sánchez García, J. y Villanueva Moya, L. (2016). ¿Satisfacción por vocación?: La influencia del tipo de trabajo en la satisfacción laboral. ReiDoCrea, 5, 90-96.

\begin{abstract}
Resumen: Actualmente se ha enfatizado la satisfacción laboral para el desempeño del individuo en su trabajo. De este modo, es importante considerar los factores que influyen en ella. En la presente investigación se pretende comprobar si existe relación entre el tipo de trabajo (ideal/real) que desempeña el individuo y la satisfacción laboral dependiendo de factores intrínsecos o extrínsecos a la persona. Se espera encontrar mayor satisfacción intrínseca en personas que desempeñan el trabajo para el que se han formado (ideal) y mayor satisfacción extrínseca en personas que desempeñan un trabajo para el cual se formaron (real). Los resultados apoyaron la primera hipótesis, pero no la segunda. Un mayor control de otros factores extrínsecos no tenidos en cuenta podría cambiar dichos resultados.
\end{abstract}

Palabras clave: Satisfacción | Motivación

Satisfaction for Vocation?: The Influence of Type of Work on Job Satisfaction

Abstract: The importance of job satisfaction for individual performance in the workplace is stressed nowadays. Thus, it is important to consider factors that affect it. The aim of the present study is to confirm if there is a relationship between the type of job (ideal/real) that an individual performs and job satisfaction depending on intrinsic or extrinsic factors. We expect to find a higher level of intrinsic satisfaction in individuals performing the job they have been trained for (ideal), and a higher level of extrinsic satisfaction in those who perform a job they have not been trained for (real). The results support our first hypothesis, but not the second. A greater control of other extrinsic factors not taken into account may change these results.

Key words: Satisfaction | Motivation

\section{Introducción}

En la construcción de un adecuado ambiente laboral, hay dos variables que se deben de tener en cuenta, las cuales son la motivación de los empleados y su satisfacción laboral. Toro (1998) establece una diferenciación entre estos conceptos y el clima organizacional indicando los efectos de estos en la productividad; mientras que la motivación se centra en la acción, la satisfacción es una consecuencia positiva de complacencia o desagrado, y el clima es un modo de percibir la realidad de forma colectiva.

La mayoría de estudios demuestran la existencia de una relación directa entre satisfacción y motivación. Respecto al trabajo, la finalidad de la conducta es obtener satisfacción, por lo que la dirección tratará de promover en el ejercicio de la práctica motivacional unas condiciones laborales que permitan conseguir satisfacción en el desempeño laboral (Castillo Clavero, 1990).

En la presente investigación, se enfatizará en el concepto de satisfacción laboral relacionándola con la motivación, resaltando las dimensiones extrínsecas e intrínsecas. Según Koenes (1996) cada persona genera un estado emocional como consecuencia de la influencia que ejercen distintos motivos en su comportamiento, siendo entendido dicho estado como motivación humana. Concretamente, este estudio se centra en la motivación laboral definida como "el nivel de esfuerzo que las personas están dispuestas a realizar en su trabajo" (Vroom, 1964). 
Distintas teorías diferencian entre factores motivacionales intrínsecos y extrínsecos, una de ellas es la teoría de la motivación-higiene (Herzberg, 1959). Atendiendo a esta teoría, entre los factores motivacionales intrínsecos destacan la realización, el crecimiento personal, el reconocimiento, aprendizaje personal y profesional, desarrollo, progreso, responsabilidad y el trabajo mismo. Por otro lado, en los factores motivacionales extrínsecos se encuentran las condiciones laborales, relaciones interpersonales, supervisión, administración, estructura y política de la compañía y el salario. Además, Herzberg (1959) informa que mientras que la motivación intrínseca no tiene contingencias externas (la realización de la conducta en sí misma es la que nos mueve) la motivación extrínseca depende de contingencias externas (motivos ajenos).

Según la literatura especializada, los factores que componen la motivación intrínseca se relacionan con la satisfacción laboral mientras que los extrínsecos evitan la insatisfacción, por otro lado es necesario tener en cuenta que la satisfacción e insatisfacción son conceptos independientes según la Teoría de motivación-higiene (Herzberg, 1959).

Además de las características personales del empleado o los rasgos de la empresa, también influye en la satisfacción laboral las propias características del empleo como, por ejemplo, el salario. Esto ha sido comprobado por la Teoría Económica (Sloane y Williams, 2000) la cual sugiere que la satisfacción laboral se vería elevada por un mayor salario y ete mayor salario dará lugar a una mayor satisfacción. Dentro de esta teoría podemos destacar la teoría microeconómica convencional (Sloane y Williams, 2000), donde se establece una relación directa entre niveles altos de salario con una alta satisfacción. Por su parte, la Teoría Económica (Sloane y Williams, 2000) sugiere que la satisfacción laboral se vería elevada por un mayor salario y además un mayor salario dará lugar a una mayor satisfacción.

Concretamente la motivación intrínseca es favorecedora para el bienestar en general, satisfacción laboral, compromiso, competencia y productividad en el trabajo, tal como reconoce la Teoría de la auto-determinación (Deci y Ryan, 1985). Esta teoría se interesa por los comportamientos, creencias y sentimientos, intentando promover la autonomía del trabajador, así como la voluntad propia, la decisión y el sentido de elección, en definitiva la competencia del trabajador.

Por su parte, la satisfacción laboral (constructo multidimensional) se define como el bienestar subjetivo que experimenta el trabajador tras su actividad laboral. Tal satisfacción dará lugar a un conjunto de respuestas afectivas que influirán en el desempeño del trabajador en su empresa. Las variables a tener en cuenta para el grado de satisfacción laboral son: el absentismo, el abandono del trabajador, y su propia productividad. En este sentido, Lévy-Garbona y Montmarquette (2001) consideran que la satisfacción laboral es una expresión expost de la preferencia que el trabajador muestra sobre el trabajo que ejerce, es decir, trabajo "real" respecto a otro que es el ideal (entendiendo como trabajo ideal aquel puesto de trabajo para el cual se ha formado la persona, según las investigadoras del presente estudio).En este sentido, la teoría de la discrepancia (Higgins, 1987) establece una diferenciación entre el trabajo real e ideal indicando que la satisfacción es el resultado de un desequilibrio entre estos dos tipos de trabajo. Este desequilibrio viene como resultado de los juicios que el empleado hace de una comparación entre ambos tipos de trabajo.

Así, el concepto principal de las distintas teorías motivacionales en el trabajo es el de satisfacción, entendido en definitiva, como una orientación afectiva, que experimenta 
el individuo, hacia su empleo y hacia sí mismo, traducida cuando es positiva en sentimientos de felicidad, placer y bienestar (Castillo Clavero, 1990).

Teniendo esto en cuenta, el objetivo del presente estudio será investigar la relación entre motivación (extrínseca e intrínseca) con el trabajo (ideal-real) y la relación entre la satisfacción laboral y el trabajo (ideal-real). Considerando la percepción de crisis de cada empleado, su tipo de contrato y su salario.

De esta manera, los resultados de anteriores investigaciones, esperamos que las personas con un trabajo ideal (trabajo para el cual se han formado) tengan mayor motivación intrínseca que las personas con un trabajo real pero no ideal, mientras que las personas con un trabajo real tendrán mayor motivación extrínseca (Hipótesis 1) que las personas con un trabajo ideal. Por otro lado, se espera que las personas con un trabajo ideal tengan una mayor satisfacción laboral (Hipótesis 2).

\section{Método}

\section{Participantes}

La presente investigación se llevó a cabo con la colaboración de 30 personas de nacionalidad española. Todos los participantes desempeñaban un trabajo en la actualidad, de los cuales $17(56,5 \%)$ indicaron estar ejerciendo su trabajo ideal y 13 $(43,3 \%)$ ejercer un trabajo que no corresponde a los estudios realizados. De la muestra, el $53,3 \%$ fueron mujeres y el $46,6 \%$ varones. Las edades estuvieron comprendidas entre 23 y 54 años, siendo la media de las edades de 40,1 (DT=11,33).

\section{Instrumentos}

Para llevar a cabo el presente estudio, se ha utilizado el cuestionario "NTP 394: Satisfacción laboral: escala general de satisfacción" (Warr, Cook y Wall, 1979), el cual consta de 15 ítems que debían que ser contestados por los participantes indicando su grado de satisfacción o insatisfacción en una escala de 1 a 7 (siendo 1 muy insatisfecho y 7 muy satisfecho). En dichos ítems se pregunta sobre aspectos relativos al reconocimiento, responsabilidad, promoción, contenido de la tarea, organización del trabajo, horario y condiciones físicas del trabajo, entre otros aspectos. El alfa de Cronbach de la escala fue de 0,79 .

Adicionalmente se incluyeron seis preguntas que permitieron obtener información más clara y concisa de los participantes sobre el salario, tipo de contrato, formación, trabajo actual y percepción de crisis.

Para facilitar la difusión del cuestionario y acceder con más facilidad a los participantes el cuestionario fue administrado vía online, a través de la página www.survio.com.

\section{Procedimiento}

En el presente estudio la variable independiente fue el tipo de trabajo (ideal o real) y la variable dependiente fue la satisfacción laboral, la cual recoge dos aspectos, satisfacción intrínseca y satisfacción extrínseca. El tipo de muestreo empleado para seleccionar la muestra fue un muestreo no probabilístico por conveniencia.

En primer lugar, se solicitó la colaboración voluntaria de los participantes mediante un consentimiento informado donde se explicaba la tarea que tenían que realizar y se aseguraba su confidencialidad. Tanto este consentimiento como el cuestionario fue 
administrado vía online. Una vez aceptada la colaboración de los participantes, se le enviaba el enlace correspondiente al cuestionario, el cual podían responder individualmente en el momento preferible por cada uno. En primer lugar se les presentaba la escala dirigida a conocer su satisfacción laboral (Escala General de Satisfacción, Warr, Cook y Wall, 1979), posteriormente contestaban a las preguntas sobre salario, tipo de contrato, formación, trabajo actual y la percepción ante la crisis. El tiempo requerido para contestar dicho cuestionario era aproximadamente de 10 minutos.

\section{Análisis de datos}

Los análisis estadísticos se han llevado a cabo con el programa SPSS (22.00), utilizándose las pruebas ANOVA univariante para realizar un contraste de medias entre grupos, y una correlación bivariada para ver la relación entre variables.

\section{Resultados}

Se ha efectuado un ANOVA univariante para comprobar el efecto del trabajo actual sobre la satisfacción general de los sujetos. Se introdujo como variable independiente el trabajo actual, y la satisfacción total fue tomada como variable dependiente. Tras analizar los resultados $[F(1,28)=4,531, p<0,05]$ se apreciaron diferencias significativas (figura 1). De esta forma, se aprecia que los sujetos que están desempeñando un trabajo ideal $(M=4,83, D T=0.77)$ tienen mayor satisfacción general que aquellos que están llevando a cabo un trabajo no ideal $(M=4,03, D T=1,27)$ (Véase Figura 1). Del mismo modo, se ha analizado el efecto del salario, tipo de contrato, y descenso social sobre la satisfacción general, sin encontrar ningún efecto $(\mathrm{F}<1)$.

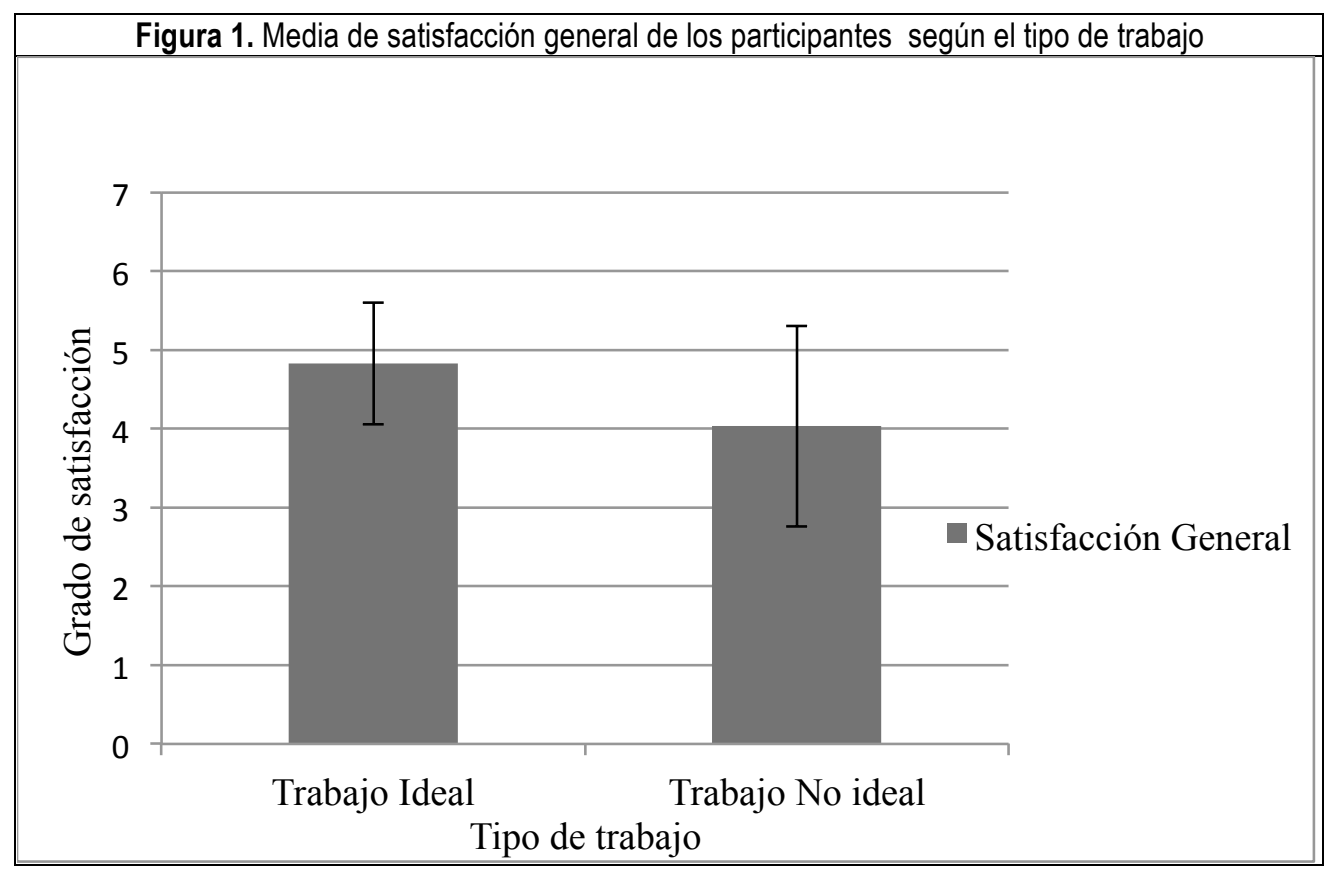

Seguidamente realizamos de nuevo el análisis anterior, tomando en este caso como variable dependiente la satisfacción intrínseca y la variable independiente el trabajo actual. Tras analizar los resultados $[F(1,28)=4,82 ; p<0,05]$ se observa que existen diferencias significativas (figura 2), en el sentido que los sujetos que están desempeñando un trabajo ideal $(M=4,99, D T=0,97)$ tienen mayor satisfacción intrínseca que aquellos que están llevando a cabo un trabajo real $(M=4,07, D T=1,33)$. 
De igual manera se ha analizado el efecto del salario, tipo de contrato, y descenso social sobre la satisfacción intrínseca, sin encontrar ningún efecto significativo $(F<1)$.

De igual forma se analizó también el efecto del tipo de trabajo sobre la satisfacción extrínseca de los sujetos, siendo la variable dependiente la satisfacción extrínseca y la variable independiente el trabajo actual. Tras analizar los resultados $[F(1,30)=2,83, p$ $=0,103]$ se encontró que no existían diferencias significativas entre ambas variables (véase Figura 2). Así mismo, se ha analizado el efecto del salario, tipo de contrato, y descenso social sobre la satisfacción extrínseca, sin encontrar ningún efecto $(F<1)$.

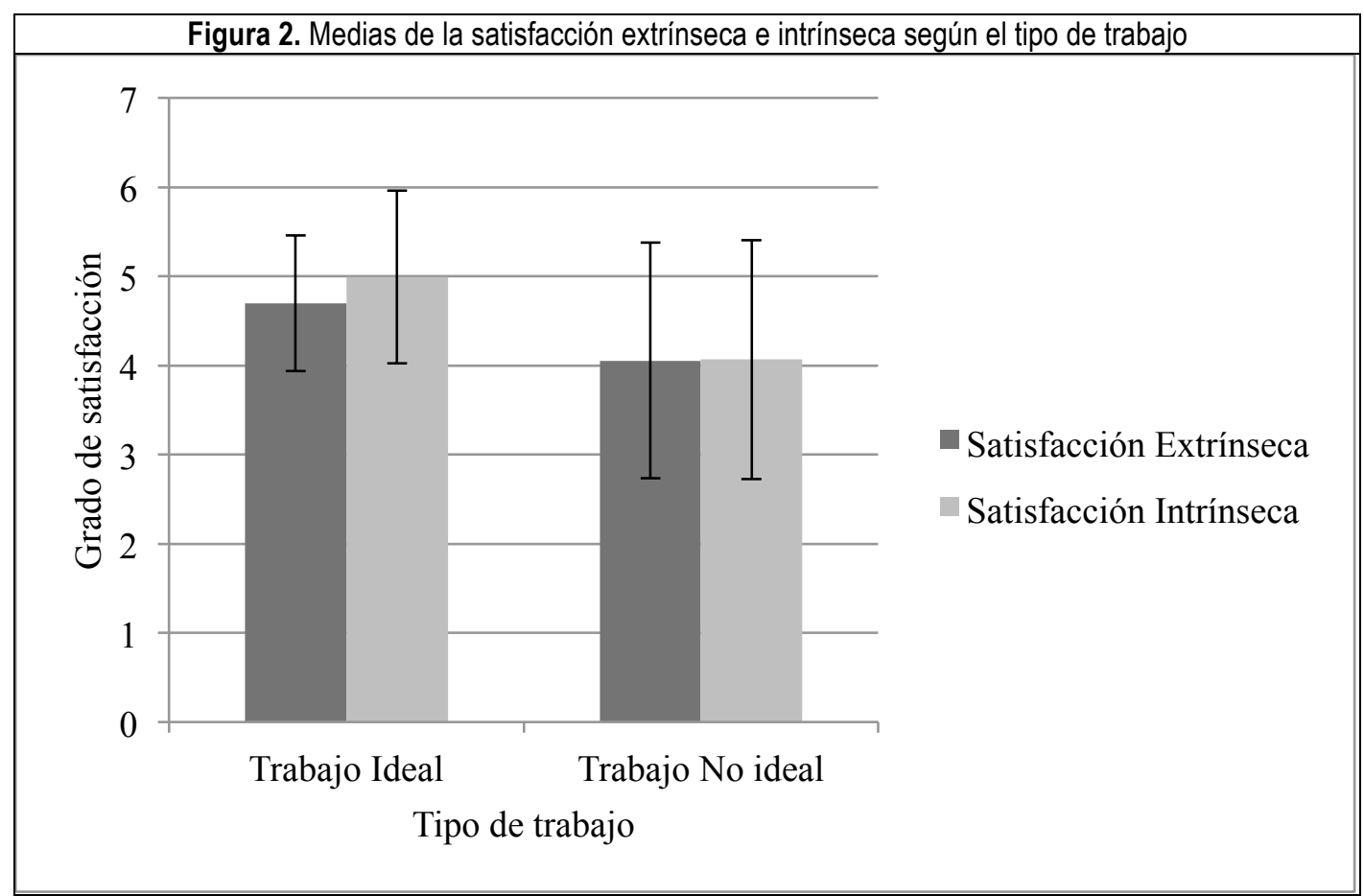

\section{Discusión}

El objetivo principal del presente estudio consistió en explorar la relación entre el tipo de trabajo que desempeñan las personas (ideal/real) y su satisfacción laboral en general, así como su satisfacción intrínseca y extrínseca. Los resultados muestran que aquellos trabajadores que están desempeñando su trabajo ideal tienen una mayor satisfacción laboral, siendo estos datos congruentes con la segunda hipótesis planteada en este estudio, la cual plantea que las personas que desempeñan su trabajo ideal presentan una mayor satisfacción laboral que aquellas que tienen un trabajo real. Estos datos son acordes a los trabajos de Higgins (1987), según el cual los trabajadores que tuvieran mayor satisfacción experimentarían un menor desequilibrio entre el trabajo desempeñado y el ideal. Por su parte, Toro (1998) afirmaba que la satisfacción es una consecuencia positiva de complacencia, por tanto, los trabajadores muy satisfechos en su trabajo tendrán un mayor desempeño en su ámbito laboral.

Concretamente, se ha encontrado una mayor satisfacción intrínseca en aquellos trabajadores que desempeñan su trabajo ideal, acorde con la primera hipótesis propuesta. Esto se debe a que el trabajo ideal es aquel para el cual se ha formado el trabajador, por lo que parece lógico pensar que se relacione con factores internos del 
individuo, y no externos. Es decir, es el trabajo en sí mismo el que produce la satisfacción y no las contingencias externas. El desempeño del trabajo ideal es lo que proporcionaría la realización, el crecimiento personal, desarrollo y responsabilidad, mencionados en la Teoría de la motivación-higiene (Herzberg, 1959). A su vez, tener un mayor grado de satisfacción intrínseca tendrá mayores consecuencias positivas en el ámbito laboral, tales como el compromiso, competencia y productividad, tal y como se propone desde la Teoría de la auto-determinación (Deci y Ryan, 1985).

Por otro lado, no se han encontrado resultados significativos entre la satisfacción extrínseca y el trabajo que desempeña actualmente el individuo (trabajo real). Tal y como afirma la Teoría de la motivación-higiene (Herzberg, 1959) esto podría deberse a que existen diferentes factores motivacionales externos al individuo que influyen en la satisfacción extrínseca, como las relaciones interpersonales, supervisión, condiciones laborales, estructura y política de la compañía y el salario.

Por su parte, la Teoría Microeconómica Convencional (Sloane y Williams, 2000) señala que altos niveles de salario tienen una relación directa con una alta satisfacción. Sin embargo, en la presente investigación se encontró que el salario no influía en gran medida en la satisfacción. Así mismo, aunque se piensa que el dinero es el principal mecanismo de recompensa y modificación conductual laboral, en realidad, apenas existe comprobación empírica que dé a conocer su uso como elemento satisfactorio. El salario por tanto, tiene un valor simbólico, ya que puede servir para satisfacer todas las necesidades, careciendo de valor intrínseco. La remuneración pues, por sí sola, puede resultar ineficiente para la satisfacción laboral. De esta manera, podría deberse a que cuando las condiciones salariales se negocian colectivamente, los sistemas de incentivos individuales pierden su papel motivador (Castillo Clavero, 1990).

En este sentido, podemos decir que para esta muestra de trabajadores si se mejorasen las condiciones laborales, la estructura de la empresa, su política y organización, y no tanto el salario, se podría observar una mayor satisfacción extrínseca y por tanto una mayor relación con el trabajo real. Por último, se ha investigado el efecto del tipo de contrato y la percepción de crisis en cuanto a la posición social del individuo en las diferentes tipos de satisfacción, no encontrándose relaciones significativas entre estos. Esto puede ser debido a que los participantes escogidos han mantenido su trabajo durante el periodo de crisis, por lo tanto, su posición social y económica no se ha visto perjudicada.

En futuras investigaciones se deberían tener en cuenta más variables extrínsecas como las mencionadas anteriormente (e.g. condiciones laborales, organización de la empresa...), además de aumentar el tamaño de la muestra. Así mismo, el muestreo debería ser más objetivo y probabilístico ya que por conveniencia podría estar sesgado.

En definitiva, si las necesidades personales hacen referencia al ser humano completo, el proceso de su satisfacción debe relacionarse con el medio ambiente total en que el individuo se desenvuelve y, del cual, el trabajo es solo una parte (Castillo Clavero, 1990).

Referencias

Alba, A. (1998). Formal training, temporary contracts, productivity and wages in Spain. Oxford Bulletin of Economics and Statistics, 56, 151-170. 
Álvarez, G. y Miles, D. (2006). El papel de la empleabilidad en la satisfacción laboral de los trabajadores temporales. Revista Galega de Economía, 15, 1-20.

Boluarte, A. (2014). Propiedades psicométricas de la Escala de satisfacción laboral de Warr, Cook y Wall, versión en español. Revista Médica Herediana, 25, 80-84.

Borra, C. y Gómez, F. (2012). Satisfacción laboral y salario: ¿Compensa la renta laboral las condiciones no monetarias del trabajo Revista de Economía Aplicada, 60, 25-51.

Bragado, M.C., Quiroga, M.C, Sánchez, M.L. y Martín, S. (2004). Autodiscrepancias y relaciones interpersonales en la adolescencia. Psicothema, 16, 182-186.

Castillo, A.M. (1990). Teoría y práctica de la motivación en la empresa. España: Gráficas Dialar.

Chaparro, L. (2006). Motivación laboral y clima organizacional en empresas de telecomunicaciones (factores diferenciadores entre las empresas pública y privada). Innovar, 16, 7-32.

Deci, E.L., \& Ryan, R.M. (1985). Intrinsic motivation and self-determination in human behavior. New York: Plemuim.

De Quijano, S. y Navarro, J. (1998). Un modelo integrado de la motivación en el trabajo: conceptualización y medida. Revista de Psicología del Trabajo y de las Organizaciones, 14, 193-216.

Gamero, C. (2007). Satisfacción laboral y tipo de contrato en España. Investigaciones Económicas, 31, 415-444.

Gamero, C. (2004). Satisfacción laboral de los asalariados en España. Especial referencia a las diferencias por género. Cuadernos de Economía, 27, 109-146.

Herzberg, F., Mausner, B., \& Snyderman, B.B. (1959). The motivation to work. Nueva York: John Wiley \& Sons.

Higgins, E.T., \& Bargh, J.A. (1987). Social cognition and social perception. Annual Review of Psychology, 38, 369-425.

Koenes, A. (1996). Gestión y motivación del personal. España: Ediciones Díaz de Santos.

Lévy-Garboua, L., \& Montmarquette, C. (2001). Satisfaction Judgments and Utility Analysis. Francia: Cirano.

Maharaj, A., Masvaure, P., \& Raggunan, S. (2014). Work engagement, intrinsic motivation and job satisfaction among employees of a diamond mining Company in Zimbabwe.Journal of Economics and Behavioral Studies, 6, 488499.

Restrepo, A. M., y Valencia, M. R. (2014). La motivación de los empleados: más allá de la "zanahoria y el garrote". Revista Ad-Minister, 24, 143-160.

Rubio, J.A.P. (1997). Motivación y satisfacción laboral: retrospectiva sobre sus formas de análisis. Reis, 80, 133-167.

Sloane, P.J., \& Williams, H. (2000). Job satisfaction, comparison earnings and gender. Labour, 14, 473-502.

Toro, F. y Cabrera H. (1998). Distinciones y relaciones entre clima, motivación, satisfacción y cultura organizacional. Revista Interamericana de Psicología Organizacional, 17, 27-39.

Vroom, V.H. (1964). Work and motivation. New Cork: Wiley

Vroom, V.H. (1979). Motivación y alta dirección. México: Trillas.

Warr, P., Cook, J., \& Wall, T. (1979). Scales for the measurement of some work attitudes and aspects of psychological well-being. Journal of Occupational Psychology, 52, 129-148. 